\title{
Table des tableaux
}

Tableau 1 Degrés d'endo- et d'exo-normativité -49

Tableau 2 Français de référence, standards nationaux et régionaux, variétés régionales - 53

Tableau 3 Approche de la norme de prononciation en linguistique - 59

Tableau 4 Inventaire vocalique du français de référence -66

Tableau 5 Auto-représentations et hétéro-représentations de l'accent/des accents parisien(s) -68

Tableau 6 Traits de prononciation appartenant potentiellement à la norme de prononciation parisienne -69

Tableau 7 Traits de prononciation appartenant potentiellement à la norme de prononciation suisse romande -74

Tableau 8 Traits de prononciation appartenant potentiellement à la norme de prononciation québécoise $-\mathbf{7 7}$

Tableau 9 Variation de la prononciation de $\langle-0 i\rangle \mid\langle-0 \hat{i}\rangle$ selon la syllabe et le lexème dans lesquels il apparaît $\longrightarrow \mathbf{7 9}$

Tableau 10 Structure du sous-chapitre sur les questionnaires $-\mathbf{9 0}$

Tableau 11 Choix des informateurs par point d'enquête selon leur sexe, leur âge et leur niveau d'éducation — 102

Tableau 12 Journalistes-présentateurs recrutés à Paris — 111

Tableau 13 Journalistes-présentateurs recrutés en Suisse romande - 112

Tableau 14 Journalistes-présentateurs recrutés au Québec — 113

Tableau 15 Traits suisses romands testés dans la liste de mots complémentaire - 116

Tableau 16 Traits québécois testés dans la liste de mots complémentaire - 116

Tableau 17 Étendue du corpus selon les régions, le média et la situation de communication -117

Tableau 18 Syntagmes du texte du programme PFC contenant les traits parisiens à analyser dans la production des locuteurs-modèles - $\mathbf{1 1 8}$

Tableau 19 Syntagmes du texte du programme PFC contenant les traits suisses romands à analyser dans la production des locuteurs-modèles - 120

Tableau 20 Syntagmes du texte du programme PFC contenant les traits québécois à analyser dans la production des locuteurs-modèles - 121

Tableau $21 \quad$ Mots tirés de la liste de mots PFC contenant les traits parisiens à analyser dans la production des locuteurs-modèles - 122

Tableau 22 Mots tirés des deux listes (liste PFC et liste complémentaire) contenant les traits suisses romands à analyser dans la production des locuteurs-modèles - $\mathbf{1 2 4}$

Tableau 23 Mots tirés des deux listes (liste PFC et liste complémentaire) contenant les traits québécois à analyser dans la production des locuteurs-modèles - 125

Tableau 24 Système de codage des voyelles nasales analysées dans le corpus - 132

Tableau 25 Stimuli pris en compte : différences entre les analyses de production et les tests de perception à l'exemple de la paire minimale patte pâte — 134

Tableau 26 Choix des stimuli -135

Tableau 27 Stimuli pris en compte dans le test de perception effectué à Paris —-137

Tableau 28 Stimuli pris en compte dans le test de perception effectué en Suisse - 138

Tableau 29 Stimuli pris en compte dans le test de perception effectué au Québec — 139 
Tableau 30

Tableau 31

Tableau 32

Tableau 33

Tableau 34

Tableau 35

Tableau 36

Tableau 37

Tableau 38

Tableau 39

Tableau 40

Tableau 41

Tableau 42

Tableau 43

Tableau 44

Tableau 45

Tableau 46

Tableau 47

Tableau 48
Questions et catégories de réponse du test de perception —-144

Taux de signification entre les six catégories prises en compte dans le modèle de régression multinomiale appliqué à la question $5-158$

Attitudes des Parisiens envers sept accents de français - $\mathbf{1 7 6}$

Précisions des informateurs suisses romands par rapport aux traits et mots les plus saillants dans leurs auto-représentations du français suisse romand $\longrightarrow \mathbf{1 8 7}$ Attitudes des Suisses romands envers différents accents du français - 214 Précisions des informateurs québécois par rapport aux traits et mots les plus saillants dans leurs auto-représentations du français québécois - 224

Répartition des réponses touchant aux régions de Québec et de Montréal à la question Selon vous, où a-t-on le moins d'accent à l'intérieur du Québec ? selon la région d'origine des informateurs (Québec, Montmagny, Montréal, Lachute) -232

Taux de signification entre les quatre catégories prises en compte dans le modèle de régression multinomiale appliqué à la question $14-247$

Attitudes des Québécois envers différents accents du français $\longrightarrow 251$

Hiérarchie de prestige des accents francophones dans les attitudes des Parisiens - 257

Prestige des accents parisiens à Paris -258

Évolution du prestige des variétés du français au Québec - 260

Moyenne et écart-type (en $\mathrm{Hz}$ ) des formants F1 et F2 mesurés dans les voyelles $/ a /$ et $/ a /$ des mots patte et $p \underline{a} t e$ chez les présentateurs parisiens et résultats du test de Wilcoxon-Mann-Whitney dans une comparaison de ces formants $(\mathrm{n}=20) \longrightarrow 266$

Moyenne et écart-type en millisecondes (ms) de la durée mesurée des voyelles / $a /$ et / $a /$ des mots p patte et pâte chez les présentateurs parisiens et résultats du test de Wilcoxon-Mann-Whitney dans une comparaison de ces durées $(n=20) \longrightarrow 267$

Types de différences (qualitatives et/ou quantitatives) (en \%) dans la réalisation de patte $\sim$ pâte chez les 20 locuteurs parisiens — 267

Moyenne et écart-type (en $\mathrm{Hz}$ ) des formants F1 et F2 mesurés dans les voyelles extraites des mots $p \underline{a}$ tte et $p \underline{a}$ tes de la lecture du texte PFC par les présentateurs parisiens et résultats du test de Wilcoxon-Mann-Whitney dans une comparaison de ces formants $(n=20)-269$

Moyenne et écart-type en millisecondes (ms) de la durée mesurée dans les voyelles des mots patte et $p \underline{a} t e$ chez les présentateurs parisiens et résultats du test de Wilcoxon-Mann-Whitney dans une comparaison de ces durées $(\mathrm{n}=20) \longrightarrow 270$

Moyenne et écart-type (en $\mathrm{Hz}$ ) des formants F1 et F2 mesurés dans les voyelles /e/ et / $\varepsilon /$ des mots épée et épais chez les présentateurs parisiens et résultats du test de Wilcoxon-Mann-Whitney dans une comparaison de ces formants $(\mathrm{n}=20)-272$

Moyenne et écart-type (en $\mathrm{Hz}$ ) des formants F1 et F2 mesurés dans les voyelles /e/ et $/ \varepsilon /$ des mots $\underline{\text { et }}$ et est extraits de la lecture du texte PFC par les présentateurs parisiens et résultats du test de Wilcoxon-Mann-Whitney dans une comparaison de ces formants $(n=20)-274$ 
Tableau 49 Moyenne et écart-type (en $\mathrm{Hz}$ ) des formants F1 et F2 mesurés dans la voyelle $/ \varepsilon /$ de étaient chez les présentateurs parisiens et résultats du test de WilcoxonMann-Whitney dans une comparaison de ces formants à ceux des voyelles /e/ dans $\underline{e t}$ et $/ \varepsilon /$ dans est de la liste de mots PFC $(n=20) \longrightarrow 275$

Tableau 50 Moyenne et écart-type (en Hz) des formants F1 et F2 mesurés dans les voyelles $/ \varnothing /$ et /œ/ des mots isolés jeûne et jeune lus par les présentateurs parisiens et résultats du test de Wilcoxon-Mann-Whitney dans une comparaison de ces formants $(n=20) \longrightarrow 277$

Tableau 51 Moyenne et écart-type (en Hz) des formants F1 et F2 mesurés dans les voyelles $/ \varnothing /$ et /œ/ des mots jeûne et jeune extraits de la lecture du texte PFC par les présentateurs parisiens et résultats du test de Wilcoxon-Mann-Whitney dans une comparaison de ces formants $(n=20) \longrightarrow \mathbf{2 8 0}$

Tableau 52 Traits appartenant potentiellement à la norme de prononciation parisienne selon les analyses acoustiques (voyelles orales) et les analyses issues des codages par accord interjuges (voyelles nasales) - 284

Tableau 53 Moyenne et écart-type en millisecondes (ms) de la durée mesurée des occurrences de /a/ et /a:/ des mots patte et pâate chez les présentateurs suisses romands et résultats du test de Wilcoxon-Mann-Whitney dans une comparaison de ces durées $(n=20)-285$

Tableau 54 Moyenne et écart-type (en $\mathrm{Hz}$ ) des formants F1 et F2 mesurés dans les occurrences de /a/ et / $a$ : / des mots patte et $p \underline{a} t e$ chez les présentateurs suisses romands et résultats du test de Wilcoxon-Mann-Whitney dans une comparaison de ces formants $(n=20)-287$

Tableau 55 Moyenne et écart-type (en $\mathrm{Hz}$ ) des formants F1 et F2 mesurés dans les voyelles extraites des mots $p \underline{a}$ tte et $p \underline{a}$ tes de la lecture du texte PFC par les présentateurs suisses romands et résultats du test de Wilcoxon-Mann-Whitney dans une comparaison de ces formants $(n=20)-288$

Tableau 56 Moyenne et écart-type (en Hz) des formants F1 et F2 mesurés dans les voyelles extraites des mots patte et $p \underline{a}$ tes de la lecture du texte PFC par les présentateurs suisses romands et résultats du test de Wilcoxon-Mann-Whitney dans une comparaison de ces formants $(n=20)-289$

Tableau 57 Moyenne et écart-type en millisecondes ( $\mathrm{ms}$ ) de la durée mesurée des occurrences de /e/ et /e:/ des mots penser et pensée chez les présentateurs suisses romands et résultats du test de Wilcoxon-Mann-Whitney dans une comparaison de ces durées $(\mathrm{n}=20) \longrightarrow 290$

Tableau 58 Moyenne et écart-type (en $\mathrm{Hz}$ ) des formants F1 et F2 mesurés à $25 \%$ et $75 \%$ des occurrences du /e:/ dans le mot pensée chez les présentateurs suisses romands et résultats du test de Wilcoxon-Mann-Whitney dans une comparaison entre ces formants $(n=20)-292$

Tableau 59 Moyenne et écart-type (en $\mathrm{Hz}$ ) des formants F1 et F2 mesurés à $25 \%$ et $75 \%$ de la voyelle /e:/ dans le mot année chez les présentateurs suisses romands et résultats du test de Wilcoxon-Mann-Whitney dans une comparaison entre ces formants $(n=20) \longrightarrow 294$

Tableau 60 Moyenne et écart-type (en Hz) des formants F1 et F2 mesurés dans les voyelles $/ e /$ et $/ \varepsilon /$ des mots pourrai et pourrais chez les présentateurs suisses romands et résultats du test de Wilcoxon-Mann-Whitney dans une comparaison de ces formants $(n=20) \longrightarrow 296$ 
Tableau 61 Traits appartenant potentiellement à la norme de prononciation suisse romande selon les analyses acoustiques (voyelles orales) et les analyses issues des codages par accord interjuges (voyelles nasales) -304

Tableau 62 Moyenne et écart-type (en $\mathrm{Hz}$ ) des formants F1 et $\mathrm{F} 2$ mesurés dans les voyelles $/ a /$ et $/ a /$ des mots patte et $p \underline{a} t e$ chez les présentateurs québécois et résultats du test de Wilcoxon-Mann-Whitney dans une comparaison de ces formants $(n=20)-306$

Tableau 63 Moyenne et écart-type en millisecondes (ms) de la durée mesurée des voyelles $/ a /$ et $/ a /$ des mots patte et $p \underline{a}$ te chez les présentateurs québécois et résultats du test de Wilcoxon-Mann-Whitney dans une comparaison de ces durées $(n=20) \longrightarrow 307$

Tableau 64 Moyenne et écart-type (en Hz) des formants F1 et F2 mesurés dans les voyelles $/ a /$ et / $a$ / extraites des mots patte et $p \underline{a} t e s$ de la lecture du texte PFC par les présentateurs québécois et résultats du test de Wilcoxon-Mann-Whitney dans une comparaison de ces formants $(n=20)-308$

Tableau 65 Moyenne et écart-type en millisecondes (ms) de la durée mesurée dans les mots patte et $p \underline{a} t e$ chez les présentateurs québécois et résultats du test de Wilcoxon-Mann-Whitney dans une comparaison de ces durées $(n=20)-308$

Tableau 66 Moyenne et écart-type (en $\mathrm{Hz}$ ) des formants F1 et F2 mesurés dans la voyelle [a] dans le mot baignoire chez les présentateurs québécois et résultats du test de Wilcoxon-Mann-Whitney dans une comparaison de ces formants à ceux des voyelles /a/ et / $a /$ dans patte et $p \underline{a}$ te de la liste de mots PFC $(n=20)-310$

Tableau 67 Moyenne et écart-type (en Hz) des formants F1 et F2 mesurés dans la voyelle [a] dans le mot trois du syntagme trois échevins chez les présentateurs québécois et résultats du test de Wilcoxon-Mann-Whitney dans une comparaison de ces formants à ceux des voyelles / $\mathrm{a} / \mathrm{et} / \mathrm{a} /$ dans patte et $p \underline{a} t e$ de la liste de mots PFC $(n=20)-312$

Tableau 68 Moyenne et écart-type en millisecondes (ms) de la durée des voyelles mesurées dans les mots faites et fête chez les présentateurs québécois et résultats du test de Wilcoxon-Mann-Whitney dans une comparaison de ces durées $(n=20) \longrightarrow 313$

Tableau 69 Moyenne et écart-type (en $\mathrm{Hz}$ ) des formants F1 et F2 mesurés à 25\% et $75 \%$ de la voyelle $/ \varepsilon$ : dans le mot fête chez les présentateurs québécois et résultats du test de Wilcoxon-Mann-Whitney dans une comparaison entre ces formants $(\mathrm{n}=20)-315$

Tableau 70 Comparaison des valeurs F1 et F2 moyennes à $25 \%$ et $75 \%$ de la voyelle $/ \varepsilon$ : chez les présentateurs de télévision de mon corpus par rapport aux locuteurs québécois « ordinaires » de Martin $(2002,86)-316$

Tableau 71 Moyenne et écart-type (en $\mathrm{Hz}$ ) des formants F1 et F2 mesurés dans les voyelles [1] et [i] des mots six $x$ et $a m \underline{i}$ chez les présentateurs québécois et résultats du test de Wilcoxon-Mann-Whitney dans une comparaison de ces formants $(n=20)-318$ 
Tableau 72 Moyenne et écart-type (en Hz) des formants F1 et F2 mesurés dans les voyelles [1] et [i] des syntagmes autour des mêmes villes et jusqu'ici les seuls titres de gloire de Beaulieu chez les présentateurs québécois et résultats du test de Wilcoxon-Mann-Whitney dans une comparaison de ces formants $(n=20) \longrightarrow 319$

Tableau 73 Traits appartenant potentiellement à la norme de prononciation québécoise selon les analyses acoustiques (voyelles orales) et les analyses issues des codages par accord interjuges (voyelles nasales) - $\mathbf{3 3 2}$

Tableau 74 Comparaison des résultats touchant aux productions et perceptions pour les quatre traits étudiés dans le cas de Paris — 363

Tableau 75 Comparaison des résultats touchant aux productions et perceptions pour les cinq traits étudiés dans le cas de la Suisse romande - 390

Tableau 76 Résumé des traits appartenant (ou non) à la norme de prononciation suisse romande - 392

Tableau 77 Comparaison des résultats touchant aux productions et perceptions pour les neuf traits étudiés dans le cas du Québec - 434

Tableau 78 Résumé schématique des traits appartenant (ou non) à la norme de prononciation québécoise -436 
